\title{
GLOBAL GEOMETRIC STRUCTURES ASSOCIATED WITH DYNAMICAL SYSTEMS ADMITTING NORMAL SHIFT OF HYPERSURFACES IN RIEMANNIAN MANIFOLDS
}

\author{
RUSLAN A. SHARIPOV
}

Received 21 January 2001 and in revised form 26 June 2001

\begin{abstract}
One of the ways of transforming hypersurfaces in Riemannian manifold is to move their points along some lines. In Bonnet construction of geodesic normal shift, these points move along geodesic lines. Normality of shift means that moving hypersurface keeps orthogonality to the trajectories of all its points. Geodesic lines correspond to the motion of free particles if the points of hypersurface are treated as physical entities obeying Newton's second law. An attempt to introduce some external force $\mathbf{F}$ acting on the points of moving hypersurface in Bonnet construction leads to the theory of dynamical systems admitting a normal shift. As appears in this theory, the force field $\mathbf{F}$ of dynamical system should satisfy some system of partial differential equations. Recently, this system of equations was integrated, and explicit formula for $\mathbf{F}$ was obtained. But this formula is local. The main goal of this paper is to reveal global geometric structures associated with local expressions for $\mathbf{F}$ given by explicit formula.
\end{abstract}

2000 Mathematics Subject Classification: 53B20, 53C15, 57R55, 53C12.

1. Introduction. Let $M$ be a Riemannian manifold of the dimension $n$. Newtonian dynamical system in $M$ is determined in local coordinates by $n$ ordinary differential equations (ODEs),

$$
\ddot{x}^{k}+\sum_{i=1}^{n} \sum_{j=1}^{n} \Gamma_{i j}^{k} \dot{x}^{i} \dot{x}^{j}=F^{k}\left(x^{1}, \ldots, x^{n}, \dot{x}^{1}, \ldots, \dot{x}^{n}\right),
$$

where $k=1, \ldots, n$. Here $\Gamma_{i j}^{k}=\Gamma_{i j}^{k}\left(x^{1}, \ldots, x^{n}\right)$ are components of metric connection, while $F^{k}$ are components of force vector $\mathbf{F}$. They determine force field of dynamical system (1.1). Let $S$ be a hypersurface in $M$ and let $p \in S$. Consider the following initial data for the system (1.1):

$$
\left.x^{k}\right|_{t=0}=x^{k}(p),\left.\quad \dot{x}^{k}\right|_{t=0}=v(p) \cdot n^{k}(p) .
$$

Here $n^{k}(p)$ are the components of the unit normal vector $\mathbf{n}$ to $S$ at the point $p$. The initial data (1.2) determine the trajectory starting from the point $p$ in the direction of the normal vector $\mathbf{n}(p)$. The quantity $v(p)$ in (1.2) is introduced to determine the modulus of initial velocity for such trajectory. 
We choose and fix some point $p_{0} \in S$, then consider a smooth function $v(p)$ defined in some neighborhood of the point $p_{0}$. Let

$$
v\left(p_{0}\right)=v_{0} \neq 0
$$

Then in some (possibly smaller) neighborhood of $p_{0}$, this function $v(p)$ does not vanish and hence takes values of some definite sign. Upon restricting $v(p)$ to such neighborhood, we use it to determine the initial velocity in (1.2). As a result, we obtain a family of trajectories of dynamical system (1.1). Displacement of points of hypersurface $S$ along these trajectories determines shift maps $f_{t}: S \rightarrow S_{t}$. Relying upon the theorem on smooth dependence on initial data for the system of ODEs (see $[15,20]$ ), we can assume that the shift maps $f_{t}: S^{\prime} \rightarrow S_{t}^{\prime}$ are defined in some neighborhood $S^{\prime}$ of the point $p_{0}$ on $S$ for all values of the parameter $t$ in some interval $(-\varepsilon,+\varepsilon)$ on the real axis $\mathbb{R}$. At the cost of further restriction of the interval $(-\varepsilon,+\varepsilon)$, we can make the maps $f_{t}: S^{\prime} \rightarrow S_{t}^{\prime}$ diffeomorphisms and make their images $S_{t}^{\prime}$ smooth hypersurfaces, disjoint union of which fills some neighborhood of the point $p_{0}$ in $M$. Moreover, at the cost of the restriction of the neighborhood $S^{\prime}$ and the range of the parameter $t$, we can reach the situation in which shift trajectories would cross hypersurfaces $S_{t}$ transversally at all points of mutual intersection. For such a case we state the following definitions.

DEFinITION 1.1. The shift $f_{t}: S^{\prime} \rightarrow S_{t}^{\prime}$ of some part $S^{\prime}$ of the hypersurface $S$ along trajectories of Newtonian dynamical system (1.1) is called a normal shift if all hypersurfaces $S_{t}^{\prime}$ arising in the process of shifting are perpendicular to the trajectories of this shift.

DeFinITION 1.2. Newtonian dynamical system (1.1) with force field $\mathbf{F}$ is called a system admitting normal shift in strong sense if for any hypersurface $S$ in $M$, for any point $p_{0} \in S$, and for any real number $v_{0} \neq 0$, we can find a neighborhood $S^{\prime}$ of the point $p_{0}$ on $S$, and a smooth function $v(p)$, which does not vanish in $S^{\prime}$ and which is normalized by condition (1.3), such that the shift $f_{t}: S^{\prime} \rightarrow S_{t}^{\prime}$, defined by this function, is a normal shift in the sense of Definition 1.1.

First, we used the definition without the normalizing condition (1.3) for the function $v(p)$. Such definition is called the normality condition. Definition 1.2 strengthens this condition making it more restrictive with respect to the choice of force field $\mathbf{F}$ of the dynamical system (1.1). Therefore it is called strong normality condition.

Definitions 1.1 and 1.2 form the base of the theory of dynamical systems admitting the normal shift. This theory was constructed in $[2,4,5,6,7,8,9,10,11,12,13$, $14,22,24,26,27,28,29,30]$. The results of these papers were used in preparing theses [3, 31].

As it was shown in [31], Newtonian dynamical systems admitting the normal shift of hypersurfaces in Riemannian manifolds of the dimension $n \geqslant 3$ can be effectively described. The force field of such systems is given by the explicit formula

$$
F_{k}=\frac{h(W) N_{k}}{W_{v}}-v \sum_{i=1}^{n} \frac{\nabla_{i} W}{W_{v}}\left(2 N^{i} N_{k}-\delta_{k}^{i}\right),
$$


which contains one arbitrary function of one variable $h=h(w)$ and one arbitrary function of $(n+1)$ variables $W=W\left(x^{1}, \ldots, x^{n}, v\right)$ restricted by the natural condition

$$
W_{v}=\frac{\partial W}{\partial v} \neq 0 .
$$

The components of the gradient $\nabla W$ in formula (1.4) are the partial derivatives

$$
\nabla_{i} W=\frac{\partial W}{\partial x^{i}}
$$

Here $N^{i}$ and $N_{k}$ are the components of the unit vector $\mathbf{N}$ directed along the velocity vector:

$$
N^{i}=\frac{v^{i}}{|\mathbf{v}|}, \quad N_{k}=\frac{v_{k}}{|\mathbf{v}|} .
$$

Note that $v$ in (1.5) and (1.6) is treated as independent variable being $(n+1)$ th argument of the function $W\left(x^{1}, \ldots, x^{n}, v\right)$. But in formula (1.4) it designates modulus of the velocity vector. Therefore, upon calculating partial derivatives and upon substituting (1.5), (1.6), and (1.7) into (1.4), the independent variable $v$ should be replaced by $|\mathbf{v}|$.

2. The problem of globalization. If we fix a pair of functions $(h, W)$, then (1.4) uniquely determines the force field $\mathbf{F}$ of Newtonian dynamical system (1.1). However, fixing force field (1.4), we cannot uniquely determine the corresponding pair of functions $(h, W)$. In particular, global force field $\mathbf{F}$ can be represented by different pairs of functions in different local maps forming an atlas of the manifold $M$. This leads to a problem of describing global geometric structures associated with such a way of defining force field F. This problem was formulated by Kozlov and Romanovsky when I was reporting my thesis [31] in the seminar of Netsvetaev at Saint-Petersburg department of Steklov Mathematical Institute December (2000).

There is another problem of globalization concerning the process of normal shift of some particular hypersurface $S$ along trajectories of dynamical system (1.4). We will call it second problem of globalization, though, historically, it arises earlier than the first one. The second problem was formulated by Mishchenko when I was reporting, thesis in the seminar of the Chair of higher geometry and topology at Moscow State University December (2000). It is expedient to deal with the second problem of globalization only upon solving the first one. Therefore we will consider it in a separate paper.

3. Some general remarks on formula (1.4) and on the theory of Newtonian dynamical systems. Our further consideration will be based mainly on formula (1.4). However, passing from Definitions 1.1 and 1.2 directly to formula (1.4), we omit a substantial amount of the theory. In this section, we sketch in brief this omitted part of the theory and characterize our approach to Newtonian dynamical systems in whole.

First of all, note that the systems of second-order ODEs describing dynamics on manifolds appear not only in Newtonian mechanics, but also in [33, 34] for example. In the general case, when the manifold is not equipped with Riemannian metric, they 
are written as

$$
\ddot{x}^{k}=\Phi\left(x^{1}, \ldots, x^{n}, \dot{x}^{1}, \ldots, \dot{x}^{n}\right), \quad k=1, \ldots, n .
$$

Equation (3.1) can be written in the form of first-order ODEs

$$
\dot{x}^{k}=v^{k}, \quad \dot{v}^{k}=\Phi\left(x^{1}, \ldots, x^{n}, v^{1}, \ldots, \dot{v}^{n}\right) .
$$

In this form they describe the dynamics in the tangent bundle $T M$ corresponding to the following vector field:

$$
\Phi=v^{1} \cdot \frac{\partial}{\partial x^{1}}+\cdots+v^{n} \cdot \frac{\partial}{\partial x^{n}}+\Phi^{1} \cdot \frac{\partial}{\partial v^{1}}+\cdots+\Phi^{n} \cdot \frac{\partial}{\partial v^{n}} .
$$

In our case, when $M$ is a Riemannian manifold, there is a canonical map identifying tangent space $T_{q}(T M)$ with direct sum of two copies of tangent space $T_{p}(M)$, where $p=\pi(q)$ and $\pi: T M \rightarrow M$ is a projection of $T M$ onto the base $M$,

$$
T_{q}(T M) \longrightarrow T_{p}(M) \oplus T_{p}(M)
$$

Applying this map to vector (3.3), we obtain two vectors in $T_{p}(M)$ : the first is the vector of velocity $\mathbf{v}=\pi_{*}(\Phi)$ represented by the formula

$$
\mathbf{v}=v^{1} \cdot \frac{\partial}{\partial x^{1}}+\cdots+v^{n} \cdot \frac{\partial}{\partial x^{n}}=\sum_{k=1}^{n} v^{k} \cdot \frac{\partial}{\partial x^{k}}
$$

and the second is the force vector $\mathbf{F}$. It is represented by the formula

$$
\mathbf{F}=\sum_{k=1}^{n}\left(\Phi^{k}+\sum_{i=1}^{n} \sum_{j=1}^{n} \Gamma_{j k}^{i} v^{i} v^{j}\right) \cdot \frac{\partial}{\partial x^{k}} .
$$

The components of this vector (3.6) are used when we write (1.1). The map (3.4) arises in various papers, in particular, it was used by Anosov in [1], which is very famous in the theory of dynamical systems.

Vectors (3.5) and (3.6) are tangent to $M$, but they depend on the point $q \in T M$. Therefore they do not form vector fields in $M$. They form sections of pullback vector bundle $\pi^{*}\left(T_{0}^{1} M\right)$ induced by the map $\pi: T M \rightarrow M$. In [2, 4, 5, 6, 7, 8, 9, 10, 11, 12, 13, $14,22,24,26,27,28,29,30]$ and in theses [3, 31], such sections are called extended vector fields in $M$, while sections of the pullback tensor bundle $\pi^{*}\left(T_{s}^{r} M\right)$ are called extended tensor fields of type $(r, s)$.

We denote by $\mathfrak{I}(T M)$ the ring of smooth functions in $T M$. The set of all smooth extended tensor fields form a graded algebra over this ring. It is called the extended algebra of tensor fields in $M$. If $M$ is a Riemannian manifold, then we can define two covariant differentiations in the extended algebra of tensor fields. The first is given by the following explicit formula in local coordinates:

$$
\begin{aligned}
\nabla_{m} X_{j_{1} \cdots j_{s}}^{i_{1} \cdots i_{r}}= & \frac{\partial X_{j_{1} \cdots j_{s}}^{i_{1} \cdots i_{r}}}{\partial x^{m}}-\sum_{a=1}^{n} \sum_{b=1}^{n} v^{a} \Gamma_{m a}^{b} \frac{\partial X_{j_{1} \cdots j_{s}}^{i_{1} \cdots i_{r}}}{\partial v^{b}} \\
& +\sum_{k=1}^{r} \sum_{a_{k}=1}^{n} \Gamma_{m a_{k}}^{i_{k}} X_{j_{1} \cdots j_{s}}^{i_{1} \cdots a_{k} \cdots i_{r}}-\sum_{k=1}^{s} \sum_{b_{k}=1}^{n} \Gamma_{m j_{k}}^{b_{k}} X_{j_{1} \cdots b_{k} \cdots j_{s}}^{i_{1} \cdots i_{r}} .
\end{aligned}
$$


The second covariant differentiation $\tilde{\nabla}$ is given by much simpler formula,

$$
\tilde{\nabla}_{m} X_{j_{1} \cdots j_{s}}^{i_{1} \cdots i_{r}}=\frac{\partial X_{j_{1} \cdots j_{s}}^{i_{1} \cdots i_{r}}}{\partial v^{m}} .
$$

Note that (3.8) does not depend on the presence of Riemannian metric in $M$. This means that covariant differentiation $\tilde{\nabla}$ is defined for arbitrary smooth manifold $M$. It is called canonical vertical covariant differentiation. It is also called velocity covariant differentiation or velocity gradient. The first covariant differentiation $\nabla$ introduced by (3.7) is called space covariant differentiation or space gradient.

As an introduction to the theory of extended tensor fields see [31, Chapters III and IV]. Slightly different way of constructing such fields is used by Sharafutdinov in [21]. But, as noted by Dairbekov, both theories are isomorphic to each other.

Now we return to strong normality condition formulated in Definition 1.2. This condition is quite transparent from a geometrical point of view, but we need an effective criterion to check if it is satisfied for a given Newtonian dynamical system. Such criterion is formulated in terms of the so-called normality equations. First the following is the system of weak normality equations:

$$
\begin{aligned}
& \sum_{i=1}^{n}\left(v^{-1} F_{i}+\sum_{j=1}^{n} \tilde{\nabla}_{i}\left(N^{j} F_{j}\right)\right) P_{k}^{i}=0, \\
& \sum_{i=1}^{n} \sum_{j=1}^{n}\left(\nabla_{i} F_{j}+\nabla_{j} F_{i}-2 v^{-2} F_{i} F_{j}\right) N^{j} P_{k}^{i} \\
& \quad+\sum_{i=1}^{n} \sum_{j=1}^{n}\left(\frac{F^{j} \tilde{\nabla}_{j} F_{i}}{v}-\sum_{r=1}^{n} \frac{N^{r} N^{j} \tilde{\nabla}_{j} F_{r}}{v} F_{i}\right) P_{k}^{i}=0
\end{aligned}
$$

that was derived in $[6,7]$. Later in $[4,5]$ additional normality equations were derived

$$
\begin{aligned}
& \sum_{i=1}^{n} \sum_{j=1}^{n} P_{\varepsilon}^{i} P_{\sigma}^{j}\left(\sum_{m=1}^{n} N^{m} \frac{F_{i} \tilde{\nabla}_{m} F_{j}}{v}-\nabla_{i} F_{j}\right) \\
& =\sum_{i=1}^{n} \sum_{j=1}^{n} P_{\varepsilon}^{i} P_{\sigma}^{j}\left(\sum_{m=1}^{n} N^{m} \frac{F_{j} \tilde{\nabla}_{m} F_{i}}{v}-\nabla_{j} F_{i}\right), \\
& \sum_{i=1}^{n} \sum_{j=1}^{n} P_{\sigma}^{j} \tilde{\nabla}_{j} F^{i} P_{i}^{\varepsilon}=\sum_{i=1}^{n} \sum_{j=1}^{n} \sum_{m=1}^{n} \frac{P_{m}^{j} \tilde{\nabla}_{j} F^{i} P_{i}^{m}}{n-1} P_{\sigma}^{\varepsilon} .
\end{aligned}
$$

Normality equations (3.9) and (3.10) are written in terms of covariant derivatives (3.7) and (3.8). Components of unit vector $\mathbf{N}$ in them are given by (1.7), while $P_{k}^{i}$ are components orthogonal projector onto the hyperplane perpendicular to the vector of velocity. They are given by the formula

$$
P_{k}^{i}=\delta_{k}^{i}-N^{i} N_{k}
$$

The relation between normality equations and Definition 1.2 is established by the following theorem proved in [31, Chapter V]. 
THEOREM 3.1. Newtonian dynamical system in Riemannian manifold $M$ admits normal shift of hypersurfaces in the sense of Definition 1.2 if and only if its force field $\mathbf{F}$ satisfies both systems of normality equations (3.9) and (3.10) at all points $q=(p, \mathbf{v})$ of the tangent bundle TM, where $v=|\mathbf{v}| \neq 0$.

The next step in exploring the structure of dynamical systems admitting normal shift of hypersurfaces was made in [12], where it was found that each solution of normality equations (3.9) and (3.10) is determined by some extended scalar field $A$ :

$$
F_{k}=A N_{k}-|\mathbf{v}| \sum_{i=1}^{n} P_{k}^{i} \tilde{\nabla}_{i} A .
$$

Formula (3.12) is called scalar ansatz. Substituting (3.12) into the normality equations (3.9) and (3.10), we reduce them to the equations for the scalar function $A=$ $A\left(x^{1}, \ldots, x^{n}, v^{1}, \ldots, v^{n}\right)$. By further efforts in [31, Chapter VII], these reduced equations were solved and formula (1.4) was derived.

4. Scalar ansatz and gauge transformations. Consider the projection of the force vector (1.4) onto the direction of the velocity vector. This projection can be calculated as a scalar product of vectors $\mathbf{F}$ and $\mathbf{N}$ :

$$
A=(\mathbf{F} \mid \mathbf{N})=\sum_{k=1}^{n} F_{k} N^{k} .
$$

Substituting (1.4) into (4.1), we get the following expression for A:

$$
A=\frac{h(W)}{W_{v}}-\frac{v}{W_{v}}(\nabla W \mid \mathbf{N}) .
$$

A very important point is that the scalar field $A$ in formulas (4.1) and (4.2) is the same field as in (3.12). Therefore force fields (1.4) can be recovered by corresponding scalar fields $A$. This recovery is given by scalar ansatz (3.12). Note that in (4.2) we apply covariant derivative (3.7) to extended scalar field $W$. But the scalar field $W$ depends on the components of the velocity vector $\mathbf{v}$ only through its dependence on $v$, where $v=|\mathbf{v}|$. For such field $W$ formula (3.7) reduces to (1.6).

Formulas (4.1) and (3.12) set up a one-to-one correspondence between vector fields F of the form (1.4) and scalar fields $A$ of the form (4.2). Formula (4.2) uniquely determines the scalar field $A$ by the pair of functions $(h, W)$. But the inverse correspondence is not univalent. This is confirmed by the existence of gauge transformations,

$$
\begin{gathered}
W\left(x^{1}, \ldots, x^{n}, v\right) \rightarrow \rho\left(W\left(x^{1}, \ldots, x^{n}, v\right)\right), \\
h(w) \longrightarrow h\left(\rho^{-1}(w)\right) \cdot \rho^{\prime}\left(\rho^{-1}(w)\right),
\end{gathered}
$$

with one arbitrary function of one variable $\rho=\rho(w)$. Transformations (4.3) change $h$ and $W$, but they do not change the scalar field $A$.

We investigate which part of information on $h$ and $W$ can be recovered by $A$. Suppose that the point $p \in M$ is fixed. The dependence of $A$ on the direction of velocity vector at the point $p$ is determined by the term $\mathbf{N}$ in the scalar product $(\nabla W \mid \mathbf{N})$. 
Therefore if we change $\mathbf{v}$ by $-\mathbf{v}$, the first summand in (4.2) remains unchanged, while the second changes in sign. Hence

$$
\frac{h(W)}{W_{v}}=\frac{A(\mathbf{v})+A(-\mathbf{v})}{2}, \quad \frac{(\nabla W \mid \mathbf{N})}{W_{v}}=\frac{A(-\mathbf{v})-A(\mathbf{v})}{2|\mathbf{v}|} .
$$

Keeping the value of $v=|\mathbf{v}|$ unchanged, we can change the direction of vector $\mathbf{N}$. This allows us to determine each component of vector $\nabla W / W_{v}$. Thus by $A$ we can recover the scalar $h(W) / W_{v}$ and the vector $\nabla W / W_{v}$.

Let $p$ be a point of the manifold $M$. Suppose that the field $A$ is determined by two pairs of functions $(h, W)$ and $(\tilde{h}, \tilde{W})$ in some neighborhood of $p$. Then

$$
\frac{h(W)}{W_{v}}=\frac{\tilde{h}(\tilde{W})}{\tilde{W}_{v}}, \quad \frac{\nabla W}{W_{v}}=\frac{\nabla \tilde{W}}{\tilde{W}_{v}} .
$$

More precisely, we should note that functions $W$ and $\tilde{W}$ are determined in some domain $U$ in a Cartesian product $M \times \mathbb{R}^{+}$, where by $\mathbb{R}^{+}$we denote the set of positive real numbers. Second relationship in (4.5) means that the complete gradients of these two functions in $U$ are collinear:

$$
\operatorname{grad} W \| \operatorname{grad} \tilde{W}
$$

The conditions $W_{v} \neq 0$ and $\tilde{W}_{v} \neq 0$ mean that both gradients in (4.6) are nonzero. This situation is described by the following lemma.

LEMMA 4.1. If the gradient of one smooth function $f\left(x^{1}, \ldots, x^{n}\right)$ is nonzero in some domain $U \subset \mathbb{R}^{n}$ and the gradient of another smooth function $g\left(x^{1}, \ldots, x^{n}\right)$ is collinear to it in $U$, then functions $f$ and $g$ are functionally dependent in $U$. This means that for each point $p \in U$, we can find some neighborhood $O(p)$ and a smooth function of one variable $\rho(y)$ such that $g=\rho \circ f$ in $O(p)$.

Lemma 4.1 is a purely local fact following from the theory of implicit functions (see $[16,18])$. But, in spite of this, it is relevant, since it describes the structure of nonuniqueness in inverse correspondence for $(h, W) \rightarrow A$.

THEOREM 4.2. Suppose that two pairs of functions $(h, W)$ and $(\tilde{h}, \tilde{W})$, defined in some domain $U \subset M \times \mathbb{R}^{+}$, determine the same force field $\mathbf{F}$ of the form (1.4). Then for each point $q \in U$, we can find some neighborhood $O(q)$ and a smooth function of one variable $\rho(y)$ such that $(h, W)$ and $(\tilde{h}, \tilde{W})$ are bound by the gauge transformation (4.3) in $O(q)$.

5. Projectivization of cotangent bundle. Denote by $M$ the Cartesian product $M \times \mathbb{R}^{+}$. Let $\mathscr{T}^{*} \mathcal{M}$ be the cotangent bundle for $\mathcal{M}$. If we take the pair of functions $h$ and $W$, which determine the force field $\mathbf{F}$ of the form (1.4), then we see that the derivatives

$$
\nabla_{1} W, \nabla_{2}, \ldots, \nabla_{n} W, W_{w}
$$

constitute the set of components of differential 1-form $\omega=d W$. The domain, where this 1 -form is defined, should not coincide with the whole manifold $M$. Hence, we have 
a local section of the bundle $\mathcal{T}^{*} \mathcal{M}$. The second summand in formula (1.4) does not contain the components of differential form $\omega$ by themselves, rather, it contains the quotients

$$
b_{i}=-\frac{\nabla_{i} W}{W_{v}}=-\frac{\omega_{i}}{\omega_{n+1}}
$$

We pass to quotients of fibers of cotangent bundle $\mathcal{T}^{*} \mathcal{M}$ by the action of multiplicative group of real numbers $\omega \rightarrow \alpha \cdot \omega$. In other words, we replace linear spaces $\mathscr{T}_{q}^{*}(\mathcal{M})$ over the points $q \in \mathcal{M}$ by corresponding projective spaces $\mathscr{P}_{q}^{*}(\mathcal{M})$. As a result we get projectivized cotangent bundle $\mathscr{P} * \mathcal{M}$. This is locally trivial bundle $\mathscr{P} * \mathcal{M}$, standard fiber of which is an $n$-dimensional projective space $\mathbb{R}^{n}$ (see the definitions in [17] or [19]).

Fibers of projective bundle $\mathscr{P} * \mathcal{M}$ are parameterized by the components of covectors $\omega$ taken up to an arbitrary numeric factor:

$$
\alpha \cdot \omega_{1}, \alpha \cdot \omega_{2}, \ldots, \alpha \cdot \omega_{n}, \alpha \cdot \omega_{n+1}
$$

If $\omega_{n+1} \neq 0$, then we can choose numeric factor $\alpha=1 / \omega_{n+1}$. Then from (5.3) we obtain $-b_{1},-b_{2}, \ldots,-b_{n}, 1$. This means that quantities $b_{i}$ from (5.2) are the local coordinates in one of the affine maps in projective fiber of the bundle $\mathscr{P}^{*} \mathcal{M}$. We turn back to the problem of globalization formulated in Section 2. From formulas (4.5) we derive the following proposition.

LEMMA 5.1. Each force field $\mathbf{F}$ of the form (1.4) determines some global section $\sigma$ of projectivized cotangent bundle $\mathscr{P} * M$.

But not all global sections of the bundle $\mathscr{P} * \mu$ can be obtained in this way. There is a restriction. The matter is that on the level of cotangent bundle $\mathcal{T}^{*} \mathcal{M}$, our section $\sigma$ in Lemma 5.1 is represented by closed differential forms $\omega$, which possibly may be defined only locally. We study how this fact is reflected on the level of the projective bundle $\mathscr{P} * \mathcal{M}$. In order to recover components of the form $\omega$ in (5.3) by $b_{1}, b_{2}, \ldots, b_{n}$, we should take a proper factor $\varphi=\omega_{n+1}$. Then

$$
\omega_{i}= \begin{cases}-b_{i} \varphi & \text { for } i=1, \ldots, n \\ \varphi & \text { for } i=n+1\end{cases}
$$

Closedness of the form $\omega$ is written in the form of the following relationships:

$$
\frac{\partial \omega_{i}}{\partial x^{j}}-\frac{\partial \omega_{j}}{\partial x^{i}}=0
$$

Here we denote $v=x^{n+1}$. This is natural, since $M=M \times \mathbb{R}^{+}$. Substituting (5.4) into (5.5), for $i \leqslant n$ and $j \leqslant n$ we get

$$
\frac{\partial b_{i}}{\partial x^{j}} \varphi+\frac{\partial \varphi}{\partial x^{j}} b_{i}=\frac{\partial b_{j}}{\partial x^{i}} \varphi+\frac{\partial \varphi}{\partial x^{i}} b_{j}
$$


From the same relationships (5.5) for the case $i \leqslant n$ and $j=n+1$ we derive

$$
\frac{\partial \varphi}{\partial x^{i}}=-\frac{\partial b_{i}}{\partial v} \varphi-\frac{\partial \varphi}{\partial v} b_{i}
$$

Now we substitute the derivatives $\partial \varphi / \partial x^{i}$ and $\partial \varphi / \partial x^{j}$, calculated according to (5.7), into (5.6). As a result we obtain the equations free of $\varphi$ :

$$
\left(\frac{\partial}{\partial x^{j}}+b_{j} \frac{\partial}{\partial v}\right) b_{i}=\left(\frac{\partial}{\partial x^{i}}+b_{i} \frac{\partial}{\partial v}\right) b_{j}
$$

Note that formulas (5.8) are already known (see [31, Chapter VII, Section 4]). However, the geometric interpretation of quantities $b_{i}$ in [31] was quite different.

LEMMA 5.2. Each force field $\mathbf{F}$ of the form (1.4) determines some global section $\sigma$ of the bundle $\mathscr{P} * \mathcal{M}$ with components satisfying (5.8).

Equation (5.8) above arises as a necessary condition for the existence of closed differential 1-form $\omega$ corresponding to the section of projective bundle $\mathscr{P}^{*} \mu$. But it is a sufficient condition for the existence of such 1-form as well (certainly, only for local existence). We prove this fact. In order to integrate (5.7) we use the auxiliary system of Pfaff equations,

$$
\frac{\partial V}{\partial x^{i}}=b_{i}\left(x^{1}, \ldots, x^{n}, V\right), \quad i=1, \ldots, n
$$

The relationships (5.8) are exactly the compatibility conditions for (5.9). Remember that the variables $x^{1}, \ldots, x^{n}, v$ are local coordinates in the manifold $M=M \times \mathbb{R}^{+}$, while the first $n$ of them are local coordinates in $M$. Fix some point $p_{0} \in M$. Without loss of generality, we can assume that local coordinates of the point $p_{0}$ are equal to zero. For compatible system of Pfaff equations (5.9) we set up the following Cauchy problem at the point $p_{0}$ :

$$
\left.V\right|_{x^{1}=\cdots=x^{n}=0}=w .
$$

Thereby we take $w>0$. The solution of Cauchy problem (5.10) for (5.9) does exist and it is unique in some neighborhood of the point $p_{0}$. It is a smooth function of coordinates $x^{1}, \ldots, x^{n}$ and parameter $w$,

$$
v=V\left(x^{1}, \ldots, x^{n}, w\right)
$$

For $x^{1}=\cdots=x^{n}=0$ due to $(5.10)$, we have $V(0, \ldots, 0, w)=w$. Therefore

$$
\left.\frac{\partial V}{\partial w}\right|_{x^{1}=\cdots=x^{n}=0}=1
$$


Consider the set of points $q=\left(p_{0}, v\right)$ in $\mathcal{M}$. They form a linear ruling in Cartesian product $\mathcal{M}=M \times \mathbb{R}^{+}$. Denote it by $l_{0}=l\left(p_{0}\right)$. Equality (5.12) means that for any point $q_{0} \in l_{0}$, there is some neighborhood of this point, where we have local coordinates $y^{1}, \ldots, y^{n}, w$ related to the initial coordinates $x^{1}, \ldots, x^{n}, v$ as

$$
\begin{aligned}
x^{i} & =y^{i}, \quad i=1, \ldots, n, \\
v & =V\left(y^{1}, \ldots, y^{n}, w\right) .
\end{aligned}
$$

Back transfer to initial coordinates is determined by the function $W\left(x^{1}, \ldots, x^{n}, v\right)$ :

$$
\begin{gathered}
y^{i}=x^{i}, \quad i=1, \ldots, n \\
w=W\left(x^{1}, \ldots, x^{n}, v\right) .
\end{gathered}
$$

Function $W\left(x^{1}, \ldots, x^{n}, v\right)$ is calculated implicitly from the relationship (5.11) considered as the equation with respect to $w$.

We use (5.13) and (5.14) to simplify (5.7). Instead of the function $\varphi\left(x^{1}, \ldots, x^{n}, v\right)$ in these equations, we introduce another function,

$$
\psi\left(y^{1}, \ldots, y^{n}, w\right)=\varphi\left(y^{1}, \ldots, y^{n}, V\left(y^{1}, \ldots, y^{n}, w\right)\right)
$$

Equation (5.7) is reduced to the following equation for function (5.15):

$$
\frac{\partial \psi}{\partial y^{i}}=-B_{i} \psi
$$

The quantities $B_{i}$ are expressed through the derivatives of the function $V$ :

$$
B_{i}=\frac{1}{Z} \frac{\partial Z}{\partial y^{i}}, \quad Z=\frac{\partial V}{\partial w}
$$

It is easy to see that (5.16) is a system of Pfaff equations, that is compatible due to (5.17). Moreover, it is explicitly integrable. General solution of (5.16) is given by the following explicit formula:

$$
\psi=\frac{C(w)}{Z\left(y^{1}, \ldots, y^{n}, w\right)} .
$$

Here $C(w)$ is an arbitrary smooth function of one variable. Now we use the local invertibility of the relationship (5.15):

$$
\varphi\left(x^{1}, \ldots, x^{n}, v\right)=\psi\left(x^{1}, \ldots, x^{n}, W\left(x^{1}, \ldots, x^{n}, v\right)\right) .
$$

From (5.18) and (5.19) we derive a general solution for the system of (5.7),

$$
\varphi=C(W) \cdot W_{v}, \quad W_{v}=\frac{\partial W}{\partial v}
$$


Similar to force field $\mathbf{F}$ in formula (1.4), it is determined by two functions $C(w)$ and $W\left(x^{1}, \ldots, x^{n}, v\right)$, the latter one satisfying condition (1.5). This coincidence is not occasional. From (5.9) and from (5.19) for $b_{i}$, we derive the relationship

$$
b_{i}=-\frac{\nabla_{i} W}{W_{v}}
$$

being of the same form as (5.2). Certainly, the function $W$ in (5.21) obtained by inverting local change of variables (5.13) should not coincide with the initial function $W$ in (5.2). The relation of these two functions is characterized by Theorem 4.2. The calculations we have just made result in the following lemma, sharpening Lemma 5.2.

LEMMA 5.3. The relationships (5.8) form a necessary and sufficient condition for global section $\sigma$ of projectivized cotangent bundle $\mathscr{P} * \mu$ given by its components $b_{1}, \ldots$, $b_{n}$ in local coordinates to be related to some force field $\mathbf{F}$ of the form (1.4).

6. Involutive distributions. Relying upon Lemmas 5.2 and 5.3, now we consider some global section $\sigma$ of projectivized cotangent bundle $\mathscr{P} * \mathcal{M}$ that satisfies (5.8). We reveal invariant meaning of these equations. For this purpose we consider the vector fields

$$
\mathbf{L}_{i}=\frac{\partial}{\partial x^{i}}+b_{i} \frac{\partial}{\partial v}, \quad i=1, \ldots, n
$$

and some differential 1-form $\omega$ with components (5.4). Values of vector fields (6.1) are linearly independent at each point of the domain, where they are defined. These values belong to the kernel of the form $\omega$ for any choice of function $\varphi$ in (5.4). Equation (5.8) is exactly the commutation conditions for vector fields (6.1):

$$
\left[\mathbf{L}_{i}, \mathbf{L}_{j}\right]=0
$$

Note that global sections of the bundle $\mathscr{P} * \mathcal{M}$ are in a one-to-one correspondence with $n$-dimensional distributions in the manifold $\mathcal{M}$, whose dimension is equal to $n+1$. Indeed, in the neighborhood of each point $q \in \mathcal{M}$, the section $\sigma$ of the bundle $\mathscr{P} * \mathcal{M}$ is determined by some 1 -form $\omega$ fixed up to a scalar factor $\varphi$. But the kernel $U=\operatorname{Ker} \omega \subset$ $\mathscr{T}_{q}(\mathcal{M})$ does not depend on this factor. Therefore we have global $n$-dimensional distribution $U=\operatorname{Ker} \sigma$. And conversely, if $n$-dimensional distribution $U$ is given, then in the neighborhood of each point $q \in M$, we have 1 -form $\omega$ such that $U=\operatorname{Ker} \omega$. The form $\omega$ defines local section of the bundle $\mathscr{P} * \mathcal{M}$ in the neighborhood of the point $q$. The fact that the form $\omega$ is determined by $U$ uniquely up to a scalar factor means that local sections of the bundle $\mathscr{P} * \mathcal{M}$ are glued into one global section $\sigma$ of this bundle.

Condition (6.2) means that the distribution $U=\operatorname{Ker} \sigma$ is involutive (see [17]). In this case, in the neighborhood of each point $q \in \mathcal{M}$ the section $\sigma$ can be represented by a closed 1-form $\omega$. We introduce the following terminology.

DeFINITION 6.1. The section $\sigma$ of projectivized cotangent bundle $\mathscr{P} * \mathcal{M}$ is called closed if the corresponding distribution $U=\operatorname{Ker} \sigma$ in $M$ is involutive. 
For the sections $\sigma$ related to force fields (1.4), the manifold $\mu$ is a Cartesian product $M \times \mathbb{R}^{+}$. In this case, we have a restriction expressed by condition (1.5). It can be written as $\omega_{n+1} \neq 0$. Therefore we have the following lemma.

LEMMA 6.2. Global section $\sigma$ of projectivized cotangent bundle $\mathscr{P} * \mathcal{M}$ with base manifold $M=M \times \mathbb{R}^{+}$satisfies condition (1.5) if and only if the corresponding distribution $U=\operatorname{Ker} \sigma$ is transversal to linear rulings of cylinder $M \times \mathbb{R}^{+}$.

For the sake of brevity we will write the condition stated in Lemma 6.2 as

$$
\operatorname{Ker} \sigma=U \nVdash \mathbb{R}^{+} .
$$

The results of Lemmas 5.2, 5.3, and 6.2 can be summarized in the following lemma.

LEMMA 6.3. Each force field $\mathbf{F}$ of the form (1.4) determines some closed global section $\sigma$ of projectivized cotangent bundle $\Phi^{*} * \mu$ over the base manifold $\mu=M \times \mathbb{R}^{+}$such that it satisfies the additional condition (6.3). And conversely, each such section of the bundle $\mathscr{P} * \mathcal{M}$ corresponds to some force field $\mathbf{F}$ of the form (1.4).

7. Normalizing vector fields. Up to now we studied only the second summand in formula (4.2). And we have found that it gives rise to geometric structures mentioned in Lemma 6.3. Now we consider first summand in (4.2). Denote by $a$ the following quotient:

$$
a=\frac{h(W)}{W_{v}} .
$$

The function $a=a\left(x^{1}, \ldots, x^{n}, v\right)$ in (7.1) is invariant with respect to gauge transformations (4.3). Due to (4.5) it can be continued through the region of overlapping of two maps, in which force field $\mathbf{F}$ is determined by two different pairs of functions $(h, W)$ and $(\tilde{h}, \tilde{W})$. But, in spite of this fact, it would be wrong to interpret $a$ as a scalar field on $\mu$. The matter is that in local coordinates, for which formula (1.4) holds, the variable $v$ plays exclusive role related with the expansion of $\mathcal{M}$ into the Cartesian product $M \times \mathbb{R}^{+}$. Due to this reason we derive differential equations for the function $a=a\left(x^{1}, \ldots, x^{n}, v\right)$. We apply one of the differential operators (6.1) to $a$. This yields that

$$
\left(\frac{\partial}{\partial x^{i}}+b_{i} \frac{\partial}{\partial v}\right) a=h^{\prime}(W) \frac{\nabla_{i} W+b_{i} W_{v}}{W_{v}}-\frac{h(W)}{W_{v}} \frac{\nabla_{i} W_{v}+b_{i} W_{v v}}{W_{v}} .
$$

If we take into account (5.2), then this relationship can be transformed into

$$
\left(\frac{\partial}{\partial x^{i}}+b_{i} \frac{\partial}{\partial v}\right) a=\frac{\partial b_{i}}{\partial v} a .
$$

Note that (7.3) are also already known (see [31, Chapter VII, Section 4]). Formula (1.4) was derived as a result of integrating (5.8) and (7.3). Following [31], we append the vector fields (6.1) by the following one:

$$
\mathbf{L}_{n+1}=a \frac{\partial}{\partial v}
$$


Equations (7.3) are equivalent to the following commutation relationships:

$$
\left[\mathbf{L}_{i}, \mathbf{L}_{n+1}\right]=0, \quad i=1, \ldots, n \text {. }
$$

Now we give invariant (coordinate-free) interpretation for the relationships (7.5). Vector fields (6.1) by themselves have no invariant interpretation. But their linear span at each point $q$ coincides with $n$-dimensional subspace $U_{q} \subset \mathcal{T}_{q}(\mathcal{M})$ defined by distribution $U=\operatorname{Ker} \sigma$. Consider one-dimensional quotient spaces,

$$
\Omega_{q}=\frac{\mathscr{T}_{q}(\mathcal{M})}{U_{q}}
$$

They are glued into one-dimensional vector bundle $\Omega \mu$ over the base manifold $\mu=$ $M \times \mathbb{R}^{+}$. Let $x^{1}, \ldots, x^{n}, v$ be local coordinates in $\mu$ not necessarily related to the structure of Cartesian product $M \times \mathbb{R}^{+}$, but such that the vector $\partial / \partial v$ is transversal to $U_{q}$. Then vectors (6.1) form the base in the subspace $U_{q}$, while elements of the quotient space (7.6) are cosets of subspace $U_{q}$ represented by vectors (7.4)

$$
a=C l_{U}\left(\frac{a \cdot \partial}{\partial v}\right)
$$

Sections of one-dimensional vector bundle $\Omega \mathcal{M}$ in such local coordinates can be associated with functions $a\left(x^{1}, \ldots, x^{n}, v\right)$ or with the vector fields

$$
\mathbf{X}=a\left(x^{1}, \ldots, x^{n}, v\right) \cdot \frac{\partial}{\partial v} .
$$

DEFINITION 7.1. Vector field $\mathbf{X}$ is called normalizing field for smooth distribution $U$ if for any vector field $\mathbf{Y}$ belonging to $U$ the commutator $[\mathbf{X}, \mathbf{Y}]$ is also in $U$.

Let $\mathbf{X}$ be normalizing vector field for involutive distribution $U$ and let $\mathbf{Y}$ be in $U$. Then $\mathbf{X}+\mathbf{Y}$ is also normalizing vector field for $U$. Thus we can define normalizing sections of the bundle $\Omega \mathcal{M}$ obtained by passing to the quotient of tangent bundle $\mathscr{T} \mathcal{M}$ by distribution $U$.

DEFINITION 7.2. Section $s$ of quotient bundle $\Omega \mathcal{M}=\mathscr{T} \mathcal{M} / U$ is called normalizing section if in the neighborhood of each point $q \in \mathcal{M}$ it is represented by some normalizing vector field for the distribution $U$.

Now we can formulate the main result of this paper, characterizing global geometric structures associated with formula (1.4) for the force field $\mathbf{F}$. The following theorem follows from all what was said above.

THEOREM 7.3. Defining Newtonian dynamical system admitting the normal shift in Riemannian manifold $M$ of the dimension $n \geqslant 3$ is equivalent to defining closed global section $\sigma$ for projectivized cotangent bundle $\mathscr{P} * M$ with base $M=M \times \mathbb{R}^{+}$, satisfying the condition $\operatorname{Ker} \sigma \nVdash \mathbb{R}^{+}$, and to defining normalizing global section $s$ for one-dimensional quotient bundle $\Omega \mathcal{M}=\mathcal{T}_{M} / U$, where $U=\operatorname{Ker} \sigma$. 
8. Integration of geometric structures. Formulating Theorem 7.3, we have made a step forward in understanding global geometry associated with formula (1.4) for the force field F. But as far as the effectiveness of calculation in coordinates is concerned, we came back to a situation, in which scalar field $A$ is expressed by the formula

$$
A=a+\sum_{i=1}^{n} b_{i} v^{i},
$$

where quantities $a$ and $b_{1}, \ldots, b_{n}$ should be found as solutions of (5.8) and (7.3). Formula (4.2) was more effective. Therefore we have a natural question: can one integrate (5.8) and (7.3) globally and find the pair of functions $(h, W)$ that would define scalar field A by formula (4.2) and force field $\mathbf{F}$ by formula (1.4) on the whole manifold $M$ ?

According to Theorem 7.3, each force field $\mathbf{F}$ of Newtonian dynamical system admitting the normal shift is related to some unique closed global section $\sigma$ of the bundle $\mathscr{P}^{*} \mathcal{M}$. If such section is generated by the closed global section $\omega$ of the cotangent bundle $\mathcal{T}^{*} \mathcal{M}$, then we can construct the function $W=W(q)$ on $M$ by integrating the 1 -form $\omega$ along the curve binding the point $q$ with some fixed point $q_{0}$ on $\mathcal{M}$ :

$$
W(q)=\int_{q_{0}}^{q} \omega .
$$

Formula (8.2) yields the function $W(q)$ that possibly can be multivalued, since the first homotopy group $\pi_{1}(\mathcal{M})$ of manifold $\mu$ can be nontrivial. This ambiguity is admissible. It can be eliminated by passing to the universal covering of $\mathcal{M}$.

Apart from $\sigma$, each force field $\mathbf{F}$ of Newtonian dynamical system admitting the normal shift determines some section of quotient bundle $\Omega M=\mathscr{T} M / U$, where $U=\operatorname{Ker} \sigma$. We use the structure of Cartesian product $M \times \mathbb{R}^{+}$of $M$. This yields the vector field $\mathbf{E}$ directed along linear rulings in $M$. If $x^{1}, \ldots, x^{n}$ are local coordinates in $M$ and if $v$ is natural variable ranging in positive semiaxis $\mathbb{R}^{+}$, then in local coordinates $x^{1}, \ldots, x^{n}, v$ in $\mu$ this field is given by formula $\mathbf{E}=\partial / \partial v$. According to Theorem 7.3, we have $U=\operatorname{Ker} \sigma \nVdash \mathbb{R}^{+}$, that is, $U \nVdash \mathbf{E}$. Therefore the section $s$ of the bundle $\Omega \mu$ can be represented by the vector field

$$
\mathbf{X}=a \cdot \mathbf{E}
$$

This representation is unique, the coefficient $a$ is a scalar field (a function) on $\mathcal{M}$. The condition that $s$ is a normalizing section with respect to $U$ in local coordinates $x^{1}, \ldots, x^{n}, v$ is expressed by (7.3) for the function $a$. It is easy to check that if $a$ satisfies (7.3), then the function $\varphi=1 / a$ satisfies (5.7). Hence, if section $s$ is nonzero at all points $q \in \mathcal{M}$, then we can use $\varphi=1 / a$ as proper integrating factor in formula (5.4) determining the components of the closed 1-form $\omega$. Contracting this form with the vector field (8.3), we get

$$
\omega(\mathbf{X})=C(\omega \otimes \mathbf{X})=1 .
$$

The section $\sigma$ of the bundle $\mathscr{P} * \mathcal{M}$ determines the 1 -form $\omega$ up to a scalar factor, formula (5.4) fixes this factor within the domain of local coordinates $x^{1}, \ldots, x^{n}, v$, 
while condition (8.4) shows that 1-forms defined locally by this procedure are glued into one global closed 1-form $\omega$. Substituting its components into (8.1), we get

$$
A=\frac{1}{\omega_{n+1}}-\sum_{i=1}^{n} \frac{\omega_{i} v^{i}}{\omega_{n+1}} .
$$

Scalar field (8.5) corresponds to the force field $\mathbf{F}$ with components

$$
F_{k}=\frac{N_{k}}{\omega_{n+1}}-v \sum_{i=1}^{n} \frac{\omega_{i}}{\omega_{n+1}}\left(2 N^{i} N_{k}-\delta_{k}^{i}\right),
$$

THEOREM 8.1. If the section $s$ of the quotient bundle $\Omega \mathcal{M}=\mathscr{T} M / U$ corresponding to the force field $\mathbf{F}$ of the Newtonian dynamical system admitting the normal shift is nonzero at all points $q \in M=M \times \mathbb{R}^{+}$, then there is a global closed 1-form $\omega$ determining F according to formula (8.6).

In [31], it was noted that if the function $h(w)$ in formula (1.4) is nonzero, then, up to the gauge transformation (4.3), we can take it identically equal to unity. There, this fact was understood as purely local. Theorem 8.1 shows that it is valid in the global situation too.

9. Absence of topological obstructions. It is well known that some geometric structures cannot be realized in manifolds with nontrivial topology. Thus, on the sphere $S^{2}$, there are no smooth vector fields without critical points, where they vanish. For geometric structures from Theorem 7.3 we have no such obstructions. Indeed, on any manifold $M$ there is a smooth function $w=w(p)$ which is not identically zero. Let $W(p, v)=w(p)+v$, where $v \in \mathbb{R}^{+}$. It is obvious that the function $W(p, v)$ on Cartesian product $M \times \mathbb{R}^{+}$satisfies condition (1.5). This function defines some global force field $\mathbf{F}$ of the form (1.4) and all geometric structures from Theorem 7.3 as well.

ACKNOWLEDGments. I am grateful to A. S. Mishchenko for the invitation and the opportunity to report the results of thesis [31] and successive papers [23, 25, 32] in his seminar at Moscow State University. I am grateful to N. Yu. Netsvetaev for the invitation and for the opportunity to report the same results in the seminar at SaintPetersburg Department of Steklov Mathematical Institute. I am grateful to all participants of both seminars mentioned above and to my colleague E. G. Neufeld from Bashkir State University for fruitful discussions, which stimulated the preparation of this paper.

This work is supported by grant from Russian Fund for Basic Research (project No. 00-01-00068, coordinator Ya. T. Sultanaev), and by grant from Academy of Sciences of the Republic Bashkortostan (coordinator N. M. Asadullin). I am grateful to these organizations for financial support.

\section{REFERENCES}

[1] D. V. Anosov, Geodesic flows on closed Riemannian manifolds with negative curvature, Proc. Steklov Inst. Math. 90 (1967), 1-235, [translated from Trudy Mat. Inst. Steklov, 3-209]. 
[2] A. Yu. Boldin, On the self-similar solutions of normality equation in two-dimensional case, preprint, 1994, http://arxiv.org/abs/patt-sol/9407002.

[3] _ , Two-dimensional dynamical systems admitting the normal shift, Thesis for the degree of candidate of sciences, Russia, 2000, http://arxiv.org/abs/ math.DG/0011134.

[4] A. Yu. Boldin, A. A. Bronnikov, V. V. Dmitrieva, and R. A. Sharipov, Complete normality conditions for dynamical systems on Riemannian manifolds, preprint, 1994, http://arxiv.org/abs/astro-ph/9405049.

[5] _ Complete normality conditions for dynamical systems on Riemannian manifolds, Teoret. Mat. Fiz. 103 (1995), no. 2, 267-275.

[6] A. Yu. Boldin, V. V. Dmitrieva, S. S. Safin, and R. A. Sharipov, Dynamical systems on Riemannian manifolds that admit normal shift, preprint, 1994, http://arxiv.org /abs/hep-th/9405021.

[7] _ Dynamical systems on Riemannian manifolds that admit normal shift, Teoret. Mat. Fiz. 103 (1995), no. 2, 256-266.

[8] A. Yu. Boldin and R. A. Sharipov, Dynamical systems accepting the normal shift, preprint No. 0001-M, 1993.

[9] _ Dynamical systems that admit normal shift, Teoret. Mat. Fiz. 97 (1993), no. 3, 386-395.

[10] _ Dynamical systems that admit normal shift, Dokl. Akad. Nauk 334 (1994), no. 2, 165-167.

[11]__ Multidimensional dynamical systems that admit normal shift, Teoret. Mat. Fiz. 100 (1994), no. 2, 264-269.

[12] - On the solution of normality equations in dimension $n \geq 3$, Algebra i Analiz 10 (1998), no. 4, 37-61.

[13] A. A. Bronnikov and R. A. Sharipov, Axially symmetric dynamical systems accepting the normal shift in $\mathbf{R}^{n}$, Integrability in Dynamical Systems (Russian), Ross. Akad. Nauk, Inst. Mat. Vychisl. Tsentrom, Ufa, 1994, pp. 62-69.

[14] V. V. Dmitrieva, On the equivalence of two forms of representation of normality equations for dynamical systems in $\mathbf{R}^{n}$, Integrability in Dynamical Systems (Russian), Ross. Akad. Nauk, Inst. Mat. Vychisl. Tsentrom, Ufa, 1994, pp. 5-16.

[15] M. V. Fedoryuk, Obyknovennye Differentsialnye Uravneniya, Nauka, Moscow, 1980 (Russian).

[16] V. A. Ilin, V. A. Sadovničiı, and B. H. Sendov, Matematicheskii Analiz, Nauka, Moscow, 1979.

[17] S. Kobayasi and K. Nomidzu, Osnovy Differentsialnoi Geometrii. Tom I, Nauka, Moscow, 1981 (Russian).

[18] L. D. Kudryavtsev, Course of Mathematical Analysis, vol. I,II, Nauka, Moscow, 1985.

[19] A. S. Mishchenko, Vektornye Rassloeniya i Ikh Primeneniya, Nauka, Moscow, 1984 (Russian).

[20] I. G. Petrovskiü, Lektsii po Teorii Obyknovennykh Differentsialnykh Uravnenii, Moskov. Gos. Univ., Moscow, 1984 (Russian).

[21] V. A. Sharafutdinov, Integral Geometry of Tensor Fields, VSP, Utrecht, 1994.

[22] R. A. Sharipov, Dynamical systems accepting normal shift in Finslerian geometry, in preparation.

[23] __ Newtonian dynamical systems admitting normal blow-up of points, preprint, 2000, http://arxiv.org/abs/math.DG/0008081.

[24] - Normality conditions and affine variations of connection on Riemannian manifolds, in preparation.

[25] _ On the solutions of weak normality equations in multidimensional case, preprint, 2000, http://arxiv.org/abs/math.DG/0012110.

[26] _ Dynamical system accepting the normal shift, Uspehi Mat. Nauk 49 (1994), no. 4, 105. 
[27]___ Higher dynamical systems accepting the normal shift, Dynamical Systems Accepting the Normal Shift, Bashkir State University, Ufa, 1994, pp. 41-65.

[28] _ Metrizability by means of conformally equivalent metric for the dynamical systems, Integrability in Dynamical Systems, (BNC UrO RAN), Ufa, 1994, Institute of Mathematics, pp. 80-90.

[29] _ The problem of the metrizability of dynamical systems that admit normal shift, Teoret. Mat. Fiz. 101 (1994), no. 1, 85-93.

[30] - Metrizability of dynamical systems by a conformally equivalent metric, Teoret. Mat. Fiz. 103 (1995), no. 2, 276-282.

[31]_ Dynamical systems admitting the normal shift, Thesis for the degree of doctor of sciences, Russia, 2000, http://arxiv.org/abs/math.DG/0002202.

[32] _ Newtonian normal shift in multidimensional Riemannian geometry, Mat. Sb. 192 (2001), no. 6, 105-144.

[33] C. Udriște, Geometric dynamics, Southeast Asian Bull. Math. 24 (2000), no. 2, 313-322.

[34] _ Geometric Dynamics, Kluwer, Dordrecht, 2000.

R. A. SHARIPOV: RABOchayA STREET 5, 450003, Ufa, Russia

E-mail address: r_sharipov@ic.bashedu.ru

URL: http://www.geocities. com/CapeCanaveral/Lab/5341/ 


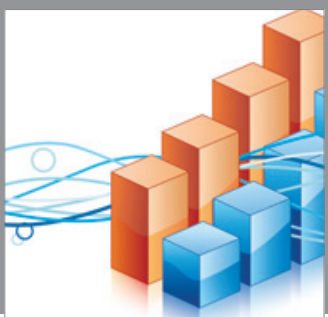

Advances in

Operations Research

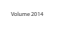

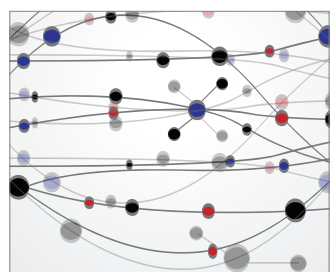

\section{The Scientific} World Journal
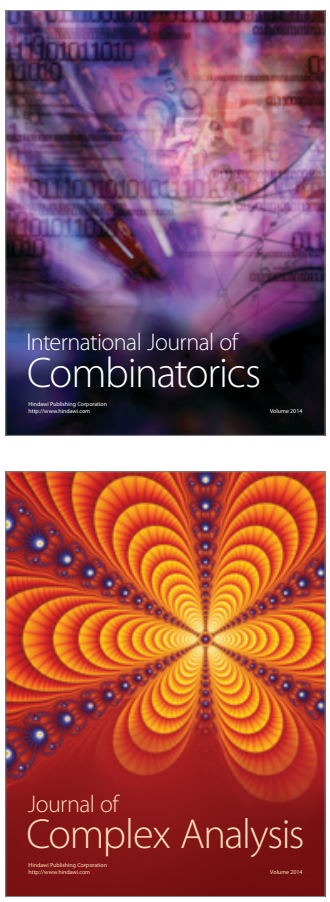

International Journal of

Mathematics and

Mathematical

Sciences
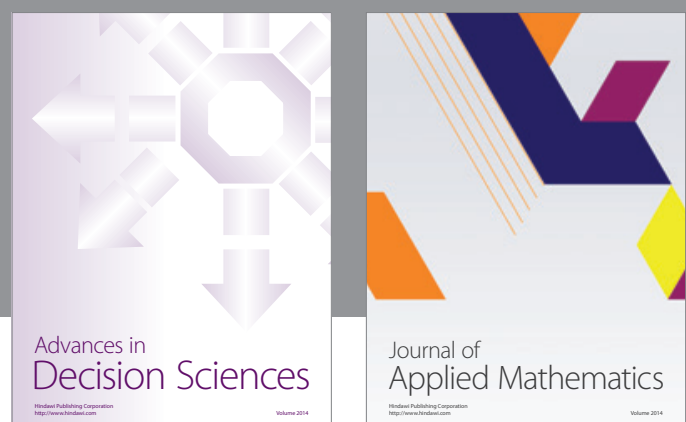

Journal of

Applied Mathematics
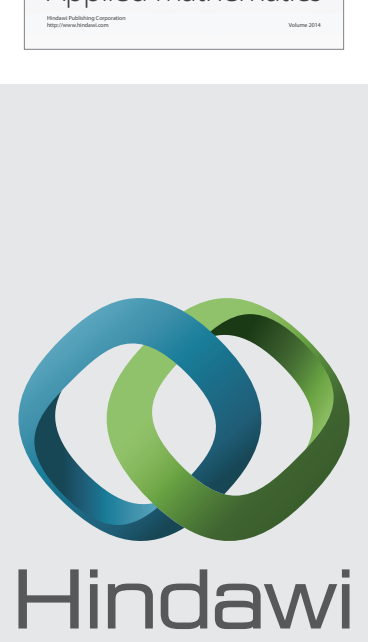

Submit your manuscripts at http://www.hindawi.com
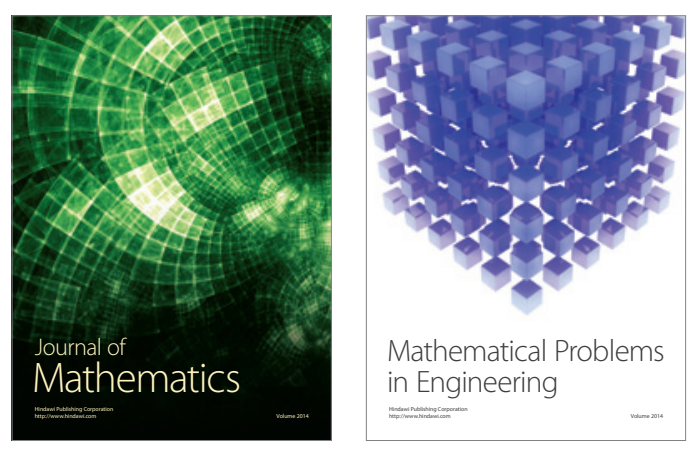

Mathematical Problems in Engineering
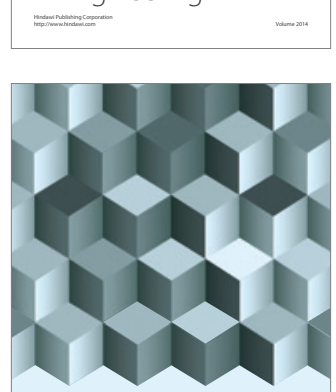

Journal of

Function Spaces
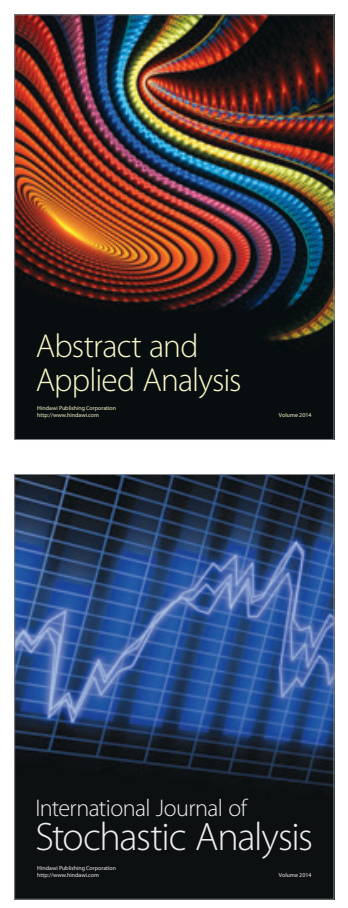

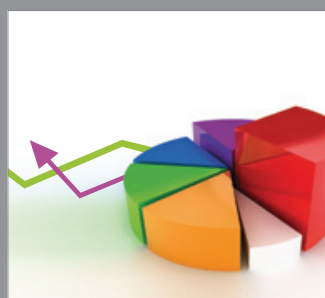

ournal of

Probability and Statistics

Promensencen
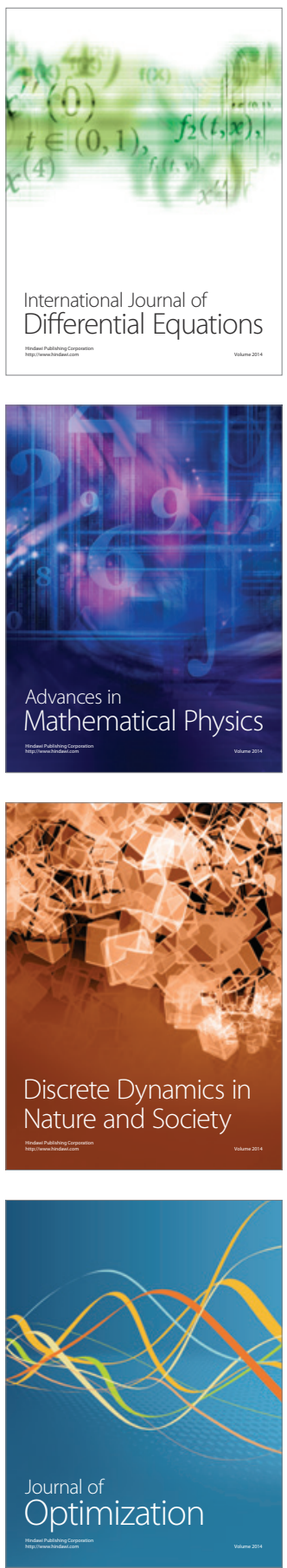